\title{
Lag synchronization of uncertain complex dynamical net- works with derivative coupling
}

\author{
Ghada Al-mahbashi*, M. S. Md Noorani \\ School of mathematical Sciences, Faculty of Science and Technology, Universiti Kebangsaan Malaysia, 43600 UKM, Selangor Darul \\ Ehsan, Malaysia.
}

\begin{abstract}
In this study, uncertain complex dynamical network model with time varying coupling delay and derivative coupling delay is considered. The lag synchronization between two such uncertain networks with different nodes is investigated. An adaptive control method is designed by using Lyapunov stability theory for achieving the lag synchronization and some corollaries are also given. In addition, on the basis of the adaptive update law, unknown parameters of the networks are estimated. The analytical results show that the states of the dynamical network with derivative delay coupling can be asymptotically synchronized under the designed control. The numerical simulation results also demonstrate the validity of the designed method.
\end{abstract}

Keywords: Lag synchronization, derivative coupling, complex dynamical networks, adaptive control.

2010 MSC: 93C40, 34D06.

(C)2019 All rights reserved.

\section{Introduction}

In recent decades, many researchers have concentrated on synchronization behavior of dynamical network, due to its ability to explain many natural phenomenons and its application in different disciplines $[3,18,20]$. When the synchronization of complex networks cannot be attained, many control schemes have been designed effectively for achieving network synchronization. In fact, after the pioneering work of Pecora and Carroll was published, many researches have been put great efforts to investigate network synchronization phenomenon $[4,6,11,12,16,17,19,21,22,29]$. In these studies, lag synchronization behavior is one of the most interesting type. This behavior has appeared in lasers, neural models, electronic applications, and secure communication [7, 13], which can cause instability and poor performance. Therefore, lag synchronization has become a hot topic in the research of complex networks and many works have been presented [1, 14, 23, 24, 31].

It should be pointed out that all the above mentioned results are concerned with the synchronization in dynamical networks when the system parameters are well known beforehand. In practical implementation, knowing the exact values of the systems parameters is difficult which may cause undesirable

\footnotetext{
${ }^{*}$ Corresponding author

Email addresses: mahbashighada@yahoo.com (Ghada Al-mahbashi), msn@ukm.my (M. S. Md Noorani)

doi: $10.22436 /$ jnsa.012.04.06
}

Received: 2018-07-22 Revised: 2018-10-05 Accepted: 2018-11-15 
dynamic behaviors and destroy the systems stability. Thus, the main motivation of this work is to study how to synchronize the networks with unknown parameters and estimate theses parameters effectively.

On the other hand, adaptive control method is an effective way to estimate the unknown parameters due to its advantages leading to stability. Therefore, it has been designed and applied effectively to explore the synchronization behavior in dynamical network with unknown parameters. In [25], exponential outer synchronization problem between two uncertain nonlinearly coupled networks with constant time delays was discussed. Ji et al. examined lag synchronization in drive-response uncertain complex dynamical network having delayed coupling and unknown parameters by adaptive control [8]. Based on a hybrid feedback control, lag synchronization in drive-response dynamical networks with non-delay coupling and unknown parameters was discussed in [15]. In [2], adaptive control method was designed to achieve the projective lag synchronization in DRDN with constant and time-varying coupling delay, also the uncertain parameters of both the network node and drive system were identified.

For a better way of describing the real world, network model should also include information of the past change rate of the nodes, such as population ecology, biologic system and ecosystem [10], where each network is shown by the present and historical fluctuating rate information. In [26], Xu et al. were the first to explore the synchronization of complex networks with derivative and non-derivative coupling. Following that pioneering work, many studies have been examined. Topological structure identification, pinning synchronization, finite-time, and pinning impulsive synchronization were studied in [5, 27, 28, 30]. In [9], Jian et al. studied the synchronization of dynamical network with time varying coupling delay and derivative coupling. To the best of our knowledge, we have not come across any theoretical results considering the problem of lag synchronization between two uncertain dynamical networks with time varying delay non-derivative and derivative coupling.

In the light of the above discussion, delayed uncertain complex dynamical network model with derivative and non-derivative coupling is proposed in this paper. The adaptive control method is developed for investigating lag synchronization and the unknown parameters are estimated. Numerical simulations results are given to prove the efficiency of the designed control.

The paper is organized as follows. Section 2 introduced the network model and some necessary preliminaries. The main results are given and novel criteria are derived in Section 3. Section 4 presented examples and their simulations. Finally, the conclusions are drawn in Section 5.

\section{Model description}

A general complex dynamical network model consisting of $\mathrm{N}$ linearly coupled nodes with uncertain parameters and delay derivative coupling can be described as

$$
\dot{x}_{i}(t)=f_{i}\left(x_{i}(t)\right)+F_{i}\left(x_{i}(t)\right) \alpha_{i}+\sum_{j=1}^{N} a_{i j} \Gamma x_{j}(t-\eta(t))+\sum_{j=1}^{N} b_{i j} \Gamma \dot{x}_{j}(t-\eta(t)),
$$

Here, $i \in \mathbf{N} \triangleq\{1,2, \ldots, N\}, N$ corresponds to the number of units in the delayed network dynamic system, $x_{i}(t)=\left(x_{i 1}(t), x_{i 2}(t), \ldots, x_{i n}(t)\right)^{\top} \in \mathbf{R}^{n}$ denotes the state vector of the $i^{\text {th }}$ node, and $f_{i}: \mathbf{R}^{n} \longrightarrow \mathbf{R}^{n}$ and $F_{i}: \mathbf{R}^{n} \longrightarrow \mathbf{R}^{n \times m_{i}}$ are the continuous nonlinear function matrices. The $\alpha_{i}$ 's are the unknown constant parameter vector, $\eta(t) \geqslant 0$ is the time varying coupling delay and $\Gamma$ is the inner coupling matrix. Also $A=\left(a_{i j}\right) \in \mathbf{R}^{N \times N}$ and $B=\left(b_{i j}\right) \in \mathbf{R}^{N \times N}$ are the coupling configuration matrices representing the coupling weights and topological structure for non delayed configuration and delayed one, where the diagonal elements of of them are defined as

$$
a_{i i}=-\sum_{j=1, j \neq i}^{N} a_{i j}, \quad b_{i i}=-\sum_{j=1, j \neq i}^{N} b_{i j} \quad i=1,2, \ldots, N
$$


We refer to model (2.1) as the drive network, and a response network is described as

$$
\dot{y}_{i}(t)=g_{i}\left(y_{i}(t)\right)+G_{i}\left(y_{i}(t)\right) \beta_{i}+\sum_{j=1}^{N} a_{i j} \Gamma y_{j}(t-\eta(t))+\sum_{j=1}^{N} b_{i j} \Gamma \dot{y}_{j}(t-\eta(t))+u_{i}(t),
$$

where $y_{i}(t)=\left(y_{i 1}(t), y_{i 2}(t), \ldots, y_{i n}(t)\right)^{\top} \in \mathbf{R}^{n}$ is response state of the $i^{\text {th }}$ node, and $g_{i}: \mathbf{R}^{n} \longrightarrow \mathbf{R}^{n}$ and $G_{i}: \mathbf{R}^{n} \longrightarrow \mathbf{R}^{n \times m_{i}}$ are the continuous nonlinear function matrices. The $\beta_{i}$ 's are the unknown constant parameter vector and $u_{i} \in \mathbf{R}^{n}$ is the control input.

Remark 2.1. Our network model has unknown parameters. Therefore, it is different from the model that was studied in [9].

Remark 2.2. Assume the model do not contain unknown parameters and $\eta(t)=0$. Then, we can get the complex network model which was when the controller $u_{i}$ can be pinning control [5], finite time control [27], and pinning impulsive control [30].

Define the lag synchronization error as

$$
e_{i}(t)=y_{i}(t)-x_{i}(t-\tau), \quad i=1, \ldots, N,
$$

where $\tau>0$ is a constant representing time delay or lag. Our objective in this paper is to design the controller $u_{i}(t)$ that makes the drive network and response network asymptotically synchronized, i.e,

$$
\lim _{t \rightarrow \infty}\left\|y_{i}(t)-x_{i}(t-\tau)\right\|=0 .
$$

Assumption 2.3 ([6]). Time delay $\eta(t)$ is a differentiable function with $0 \leqslant \dot{\eta}(t) \leqslant \varepsilon<1$. Clearly, this assumption is certainly ensured if the coupling delay $\eta(\mathrm{t})$ is a constant.

Lemma 2.4 ([16]). For any vector $x, y \in \mathbf{R}^{\mathrm{n}}$ and positive definite matrix $\mathrm{Q} \in \mathbf{R}^{\mathrm{n} \times \mathrm{n}}$, the following matrix inequality holds,

$$
2 x^{\top} y \leqslant x^{\top} Q x+y^{\top} Q^{-1} y
$$

\section{Main results}

In this section, we design an adaptive control method for achieving lag synchronization between two uncertain complex dynamical networks with time varying delayed coupling and derivative delayed coupling.

According to the networks (2.1) and (2.2), the error dynamical network for lag synchronization can be obtained as following:

$$
\begin{aligned}
\dot{e}_{i}(t)= & g_{i}\left(y_{i}(t)\right)+G_{i}\left(y_{i}(t)\right) \beta_{i}+\sum_{j=1}^{N} a_{i j} \Gamma e_{j}(t-\eta(t))+\sum_{j=1}^{N} b_{i j} \Gamma \dot{e}_{j}(t-\eta(t)) \\
& -\left(f_{i}\left(x_{i}(t-\tau)\right)+F_{i}\left(x_{i}(t-\tau)\right) \alpha_{i}\right)+u_{i}(t) .
\end{aligned}
$$

Theorem 3.1. Suppose that Assumption (2.3) holds. If there exist positive constant $\tau$, the drive network and response network can be achieve lag synchronization by using the following controllers

$$
\begin{aligned}
u_{i}(t)= & f_{i}\left(x_{i}(t-\tau)\right)+F_{i}\left(x_{i}(t-\tau)\right) \hat{\alpha}_{i}(t)-g_{i}\left(y_{i}(t)\right)-G_{i}\left(y_{i}(t)\right) \hat{\beta}_{i}(t)-\omega_{i}(t) e_{i}(t) \\
& -\sum_{j=1}^{N} r_{i}(t) \Gamma \dot{e}_{j}(t-\eta(t)), \\
\dot{\hat{\alpha}}_{i}(t)= & -\kappa_{1} F_{i}^{\top}\left(x_{i}(t-\tau)\right) e_{i}(t),
\end{aligned}
$$




$$
\begin{aligned}
\dot{\hat{\beta}}_{i}(t) & =\kappa_{2} G_{i}^{\top}\left(y_{i}(t)\right) e_{i}(t), \\
\dot{\omega}_{i}(t) & =\kappa_{3} e_{i}^{\top}(t) e_{i}(t), \\
\dot{r}_{i}(t) & =\kappa_{4} e_{i}^{\top}(t) \Gamma \dot{e}_{i}(t-\eta(t)),
\end{aligned}
$$

where $\mathrm{K}_{1}, \mathrm{~K}_{2}, \mathrm{~K}_{3}$, and $\mathrm{k}_{4}$ are positive constants. Here $\hat{\alpha}_{i}(\mathrm{t})$ and $\hat{\beta}_{i}(\mathrm{t})$ are the estimated parameters for the drive network (2.1) and response network (2.2), respectively.

Proof. Select a Lyapunov function candidate as

$$
\begin{aligned}
V(t)= & \frac{1}{2} \sum_{i=1}^{N} e_{i}^{\top}(t) e_{i}(t)+\frac{1}{2 \kappa_{1}} \sum_{i=1}^{N} \tilde{\alpha}_{i}^{\top}(t) \tilde{\alpha}_{i}(t)+\frac{1}{2 \kappa_{2}} \sum_{i=1}^{N} \tilde{\beta}_{i}^{\top}(t) \tilde{\beta}_{i}(t)+\frac{1}{2 \kappa_{3}} \sum_{i=1}^{N}\left(\omega_{i}(t)-\omega_{i}^{*}\right)^{2} \\
& +\frac{1}{2 \kappa_{4}} \sum_{i=1}^{N}\left(r_{i}(t)-\sum_{i=1}^{N} b_{i j}\right)^{2}+\frac{1}{2(1-\varepsilon)} \int_{t-\eta(t)}^{t} \sum_{i=1}^{N} e_{i}^{\top}(s) e_{i}(s) d s,
\end{aligned}
$$

where $\tilde{\alpha}_{i}(t)=\hat{\alpha}_{i}(t)-\alpha, \tilde{\beta}_{\mathfrak{i}}(t)=\hat{\beta}_{\mathfrak{i}}(t)-\beta_{i}$, and $\omega_{i}^{*}$ is positive constant.

The time derivative of $V$ is obtained as

$$
\begin{aligned}
\dot{V}= & \sum_{i=1}^{N} e_{i}^{\top}(t) \dot{e}_{i}(t)+\frac{1}{\kappa_{1}} \sum_{i=1}^{N} \dot{\hat{\alpha}}_{i}^{\top}(t) \tilde{\alpha}_{i}(t)+\frac{1}{\kappa_{2}} \sum_{i=1}^{N} \dot{\hat{\beta}}_{i}^{\top}(t) \tilde{\beta}_{i}(t)+\frac{1}{\kappa_{3}} \sum_{i=1}^{N}\left(\omega_{i}(t)-\omega_{i}^{*}\right) \dot{\omega}_{i}(t) \\
& +\frac{1}{\kappa_{4}} \sum_{i=1}^{N}\left(r_{i}(t)-\sum_{i=1}^{N} b_{i j}\right) \dot{r}_{i}(t)+\frac{1}{2(1-\varepsilon)} \sum_{i=1}^{N} e_{i}^{\top}(t) e_{i}(t) \\
& -\frac{1-\dot{\eta}(t)}{2(1-\varepsilon)} \sum_{i=1}^{N} e_{i}^{\top}(t-\eta(t)) e_{i}(t-\eta(t)) .
\end{aligned}
$$

Apply of the control function (3.2) to error dynamics (3.1), we obtain

$$
\begin{aligned}
\dot{V}= & \sum_{i=1}^{N} e_{i}^{\top}(t)\left(F_{i}\left(x_{i}(t-\tau)\right) \tilde{\alpha}_{i}(t)-G_{i}\left(y_{i}(t)\right) \tilde{\beta}_{i}(t)+\sum_{j=1}^{N} a_{i j} \Gamma e_{j}(t-\eta(t))\right. \\
& \left.+\sum_{j=1}^{N} b_{i j} \Gamma \dot{e}_{j}(t-\eta(t))-\omega_{i}(t) e_{i}(t)-\sum_{j=1}^{N} r_{i}(t) \Gamma \dot{e}_{j}(t-\eta(t))\right) \\
& +\frac{1}{\kappa_{1}} \sum_{i=1}^{N} \dot{\hat{\alpha}}_{i}^{\top}(t) \tilde{\alpha}_{i}(t)+\frac{1}{\kappa_{2}} \sum_{i=1}^{N} \dot{\hat{\beta}}_{i}^{\top}(t) \tilde{\beta}_{i}(t)+\frac{1}{\kappa_{3}} \sum_{i=1}^{N}\left(\omega_{i}(t)-\omega_{i}^{*}\right) \dot{\omega}_{i}(t) \\
& +\frac{1}{\kappa_{4}} \sum_{i=1}^{N}\left(r_{i}(t)-\sum_{i=1}^{N} b_{i j}\right) \dot{r}_{i}(t)+\frac{1}{2(1-\varepsilon)} \sum_{i=1}^{N} e_{i}^{\top}(t) e_{i}(t) \\
& -\frac{1-\eta(t)}{2(1-\varepsilon)} \sum_{i=1}^{N} e_{i}^{\top}(t-\eta(t)) e_{i}(t-\eta(t)) .
\end{aligned}
$$

By the adaptation updating laws (3.3)-(3.6), we have

$$
\begin{aligned}
\dot{V}= & \sum_{i=1}^{N} e_{i}^{\top}(t)+\sum_{j=1}^{N} a_{i j} \Gamma e_{j}(t-\eta(t))-\omega_{i}^{*} \sum_{i=1}^{N} e_{i}^{\top}(t) e_{i}(t) \\
& +\frac{1}{2(1-\varepsilon)} \sum_{i=1}^{N} e_{i}^{\top}(t) e_{i}(t)-\frac{1-\dot{\eta}(t)}{2(1-\varepsilon)} \sum_{i=1}^{N} e_{i}^{\top}(t-\eta(t)) e_{i}(t-\eta(t)) .
\end{aligned}
$$


Let us define $\Omega^{*}=\operatorname{diag}\left(\omega_{1}^{*}, \omega_{2}^{*}, \ldots, \omega_{N}^{*}\right), P=(A \otimes \Gamma), e(t)=\left(e_{1}^{\top}(t), e_{2}^{\top}(t), \ldots, e_{N}^{\top}(t)\right)^{\top}$. Then we have

$$
\dot{V}=-e^{\top}(t) \Omega^{*} e(t)+e(t)^{\top} P e(t-\eta(t))+\frac{1}{2(1-\varepsilon)} e^{\top}(t) e(t)-\frac{1-\dot{\eta}(t)}{2(1-\varepsilon)} e^{\top}(t-\eta(t)) e(t-\eta(t)) .
$$

Using Lemma (2.4), we have

$$
\begin{aligned}
\dot{V} \leqslant & -e^{\top}(t) \Omega^{*} e(t)+\frac{1}{2} e(t)^{T} P P^{\top} e(t)+\frac{1}{2} e^{\top}(t-\eta(t)) e(t-\eta(t))+\frac{1}{2(1-\varepsilon)} e^{\top}(t) e(t) \\
& -\frac{1-\dot{\eta}(t)}{2(1-\varepsilon)} e^{\top}(t-\eta(t)) e(t-\eta(t)) .
\end{aligned}
$$

From Assumption (2.3), we get

$$
\frac{1-\dot{\eta}(t)}{2(1-\varepsilon)} \geqslant \frac{1}{2}
$$

Thus, we obtain

$$
\dot{V} \leqslant e^{\top}(t)\left(\frac{1}{2} P P^{\top}+\frac{1}{2(1-\varepsilon)}-\Omega^{*}\right) e(t) .
$$

Taking $\Omega_{\mathfrak{i}}^{*}=\frac{1}{2} \mathrm{PP}^{\top}+\frac{1}{2(1-\varepsilon)}+1$, we obtain

$$
\dot{V} \leqslant-e(t)^{\top} e(t) .
$$

Based on Lyapunov stability theory, the error dynamics $e_{i}(t) \rightarrow 0$ as $t \rightarrow \infty$. That means the drive network (2.1) and response network (2.2) with delay derivative coupling achieve lag synchronization and the unknown parameters can be successfully estimated via adaptive control (3.2) and updating laws (3.3)-(3.6).

Remark 3.2. When the model does not contain derivative coupling and $\eta(t)$ is constant, then lag synchronization between uncertain drive-response complex dynamical network with non-delay coupling was discussed in Ji et al. [8] by adaptive control.

Corollary 3.3. For any given positive propagation delay $\tau$, if $\eta(t)=\eta$, then the two networks can achieve lag synchronization under the following controllers

$$
\begin{aligned}
u_{i}(t)= & f_{i}\left(x_{i}(t-\tau)\right)+F_{i}\left(x_{i}(t-\tau)\right) \hat{\alpha}_{i}(t)-g_{i}\left(y_{i}(t)\right)-G_{i}\left(y_{i}(t)\right) \hat{\beta}_{i}(t)-\omega_{i}(t) e_{i}(t) \\
& -\sum_{j=1}^{N} r_{i}(t) \Gamma \dot{e}_{j}(t-\eta), \\
\dot{\hat{\alpha}}_{i}(t)= & -\kappa_{1} F_{i}^{\top}\left(x_{i}(t-\tau)\right) e_{i}(t), \\
\dot{\hat{\beta}}_{i}(t)= & \kappa_{2} G_{i}^{\top}\left(y_{i}(t)\right) e_{i}(t), \\
\dot{\omega}_{i}(t)= & \kappa_{3} e_{i}^{\top}(t) e_{i}(t), \\
\dot{r}_{i}(t)= & \kappa_{4} e_{i}^{\top}(t) \Gamma \dot{e}_{i}(t-\eta) .
\end{aligned}
$$

Corollary 3.4. For any given positive delay $\tau$, if $A=0$ then the two networks can achieve lag synchronization under the following controllers

$$
\begin{aligned}
u_{i}(t) & =f_{i}\left(x_{i}(t-\tau)\right)+F_{i}\left(x_{i}(t-\tau)\right) \hat{\alpha}_{i}(t)-g_{i}\left(y_{i}(t)\right)-G_{i}\left(y_{i}(t)\right) \hat{\beta}_{i}(t)-\sum_{j=1}^{N} r_{i}(t) \Gamma \dot{e}_{j}(t-\eta(t)), \\
\dot{\hat{\alpha}}_{i}(t) & =-\kappa_{1} F_{i}^{\top}\left(x_{i}(t-\tau)\right) e_{i}(t), \\
\dot{\hat{\beta}}_{i}(t) & =\kappa_{2} G_{i}^{\top}\left(y_{i}(t)\right) e_{i}(t), \\
\dot{r}_{i}(t) & =\kappa_{4} e_{i}^{\top}(t) \Gamma \dot{e}_{i}(t-\eta(t)) .
\end{aligned}
$$




\section{Numerical analysis}

In this section, numerical examples are given to show the effective of the designed control methods obtained in the previous section. In the numerical simulations, the node of the drive dynamical equations are taken as the Lü chaotic system, which is given by

$$
\dot{x}_{1}(t)=\alpha_{1}\left(x_{2}(t)-x_{1}(t)\right), \quad \dot{x}_{2}(t)=\alpha_{3} x_{2}(t)-x_{1}(t) x_{3}(t), \quad \dot{x}_{3}(t)=x_{1}(t) x_{2}(t)-\alpha_{2} x_{3}(t) .
$$

The node of the response dynamics described by the following Chen chaotic system

$$
\dot{y}_{1}(t)=\beta_{1}\left(y_{2}(t)-y_{1}(t)\right), \quad \dot{y}_{2}(t)=\left(\beta_{3}-\beta_{1}\right) y_{1}(t)+\beta_{3} y_{2}(t)-y_{1}(t) y_{3}(t), \quad \dot{y}_{3}(t)=y_{1}(t) y_{2}(t)-\beta_{2} x_{3}(t),
$$

where the unknown parameter vectors are $\alpha_{i}=\left[\begin{array}{lll}\alpha_{1} & \alpha_{2} & \alpha_{3}\end{array}\right]^{\top}=\left[\begin{array}{lll}36 & 3 & 20\end{array}\right]^{\top}, \beta_{i}=\left[\begin{array}{lll}\beta_{1} & \beta_{2} & \beta_{3}\end{array}\right]^{\top}=\left[\begin{array}{lll}35 & 3 & 28\end{array}\right]^{\top}$. We take the propagation delay as $\tau=1$, the inner coupling matrix $\Gamma$ as the identity matrix and the outer coupling matrices as following:

$$
\begin{gathered}
\mathrm{A}=\left(\begin{array}{cccccccccc}
-7 & 1 & 0 & 0 & 2 & 0 & 1 & 0 & 2 & 1 \\
0 & -4 & 1 & 1 & 0 & 1 & 0 & 1 & 0 & 0 \\
1 & 0 & -5 & 0 & 1 & 0 & 2 & 0 & 1 & 0 \\
0 & 0 & 1 & -4 & 1 & 1 & 0 & 0 & 0 & 1 \\
1 & 1 & 1 & 0 & -4 & 1 & 0 & 0 & 0 & 0 \\
0 & 1 & 0 & 1 & 0 & -3 & 0 & 1 & 0 & 0 \\
0 & 0 & 0 & 0 & 0 & 1 & -2 & 0 & 1 & 0 \\
0 & 1 & 0 & 1 & 0 & 0 & 1 & -4 & 0 & 1 \\
2 & 0 & 1 & 1 & 0 & 1 & 0 & 2 & -8 & 1 \\
1 & 0 & 0 & 0 & 0 & 1 & 0 & 0 & 0 & -2
\end{array}\right), \\
\mathrm{B}==\left(\begin{array}{cccccccccc}
-4 & 1 & 1 & 0 & 0 & 0 & 1 & 0 & 0 & 1 \\
1 & -5 & 1 & 1 & 0 & 0 & 0 & 0 & 1 & 1 \\
1 & 1 & -6 & 1 & 1 & 1 & 0 & 1 & 0 & 0 \\
0 & 1 & 1 & -4 & 1 & 1 & 0 & 0 & 0 & 0 \\
1 & 0 & 1 & 1 & -7 & 1 & 1 & 1 & 0 & 1 \\
0 & 0 & 1 & 1 & 1 & -5 & 1 & 1 & 0 & 0 \\
1 & 0 & 0 & 0 & 1 & 1 & -6 & 1 & 1 & 1 \\
0 & 0 & 0 & 0 & 1 & 1 & 1 & -5 & 1 & 1 \\
0 & 1 & 0 & 0 & 0 & 0 & 1 & 1 & -4 & 1 \\
1 & 1 & 0 & 0 & 0 & 0 & 1 & 1 & 1 & -5
\end{array}\right) .
\end{gathered}
$$

According to Theorem 3.1, the adaptive laws gains are $\kappa_{1}=\kappa_{2}=\kappa_{3}=1$ and $\kappa_{4}=0.3$. The time-varying coupling delay is chosen as $\eta(t)=\frac{\exp (t)}{2(1+\exp (t))}$, then $\dot{\eta}(t)=\frac{\exp (t)}{2(1+\exp (t))^{2}} \in\left(0, \frac{1}{2}\right]$, where the Assumption 2.3 holds. The initial values are $\hat{\alpha}_{i}=\hat{\beta}_{i}=0, \omega_{i}=2, r_{i}=3$. We take the initial states as $x_{i}(0)$ and $y_{i}(0)$ are randomly chosen. The numerical results are presented in Fig. 1 and Fig. 2. The lag synchronization error is depicted in Fig. 1, showing that the lag synchronization between the drive and response networks is achieved. Fig. 2 ((a) and (b)) shows the identification of the uncertain parameters $\tilde{\alpha}$ and $\tilde{\beta}$ converge to their real values, which means that the unknown parameters are successfully estimated. These results prove the effectiveness of our designed control (3.2) with adaptive law (3.3)-(3.6) for uncertain complex dynamical networks with time varying delay coupling and delay derivative coupling.

According to corollary 3.3, when the delay coupling is constant, we choose $\eta=0.1$ and the adaptive laws gains are $\kappa_{1}=\kappa_{2}=4, \kappa_{3}=1$ and $\kappa_{4}=0.3$. The initial values are $\hat{\alpha}_{i}=\hat{\beta}_{i}=0, \omega_{i}=4, r_{i}=$ 3. We take the initial states as $x_{i}(0)$ and $y_{i}(0)$ are chosen randomly. In numerical simulation, the lag synchronization error is depicted in Fig. 3, which displays $e \longrightarrow 0$ with $t \longrightarrow \infty$. That means the required lag synchronization has been achieved with our designed control (3.7). The estimated parameters of 
the drive network nodes and response network nodes are shown in Fig. 4 ((a) and (b)) respectively, successfully estimated. These results prove the effectiveness of our designed control (3.7) with adaptive law (3.8)-(3.11) for uncertain complex dynamical networks with constant delay coupling and derivative coupling.

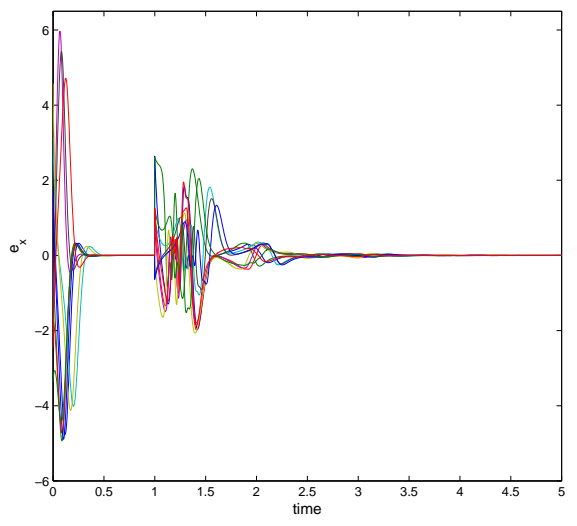

(a)

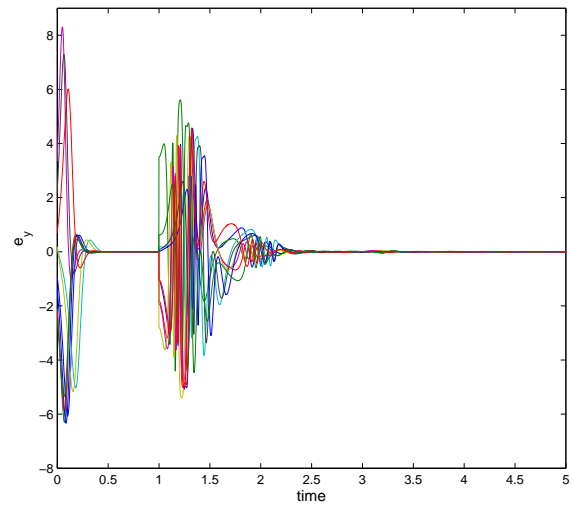

(b)

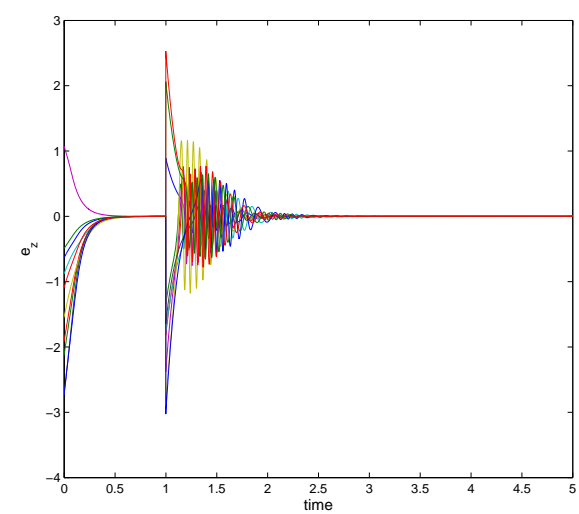

(c)

Figure 1: Time evolution of the lag synchronization error with time varying delay.

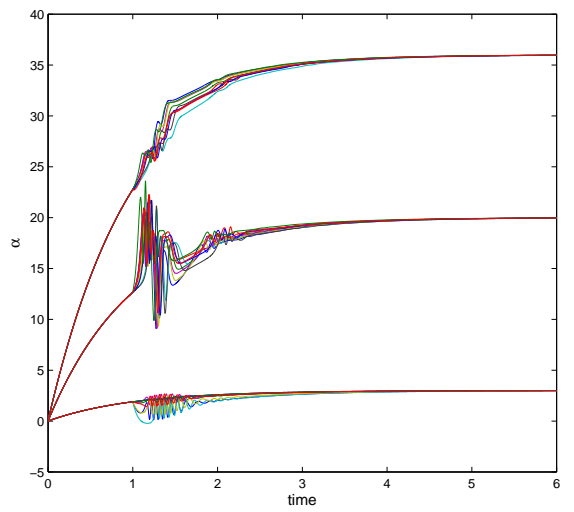

(a)

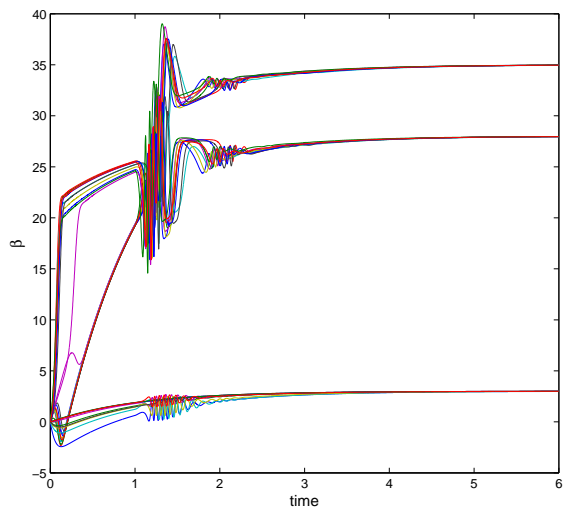

(b)

Figure 2: The estimated unknown parameter of (a) $\hat{\alpha},(b) \hat{\beta}$. 


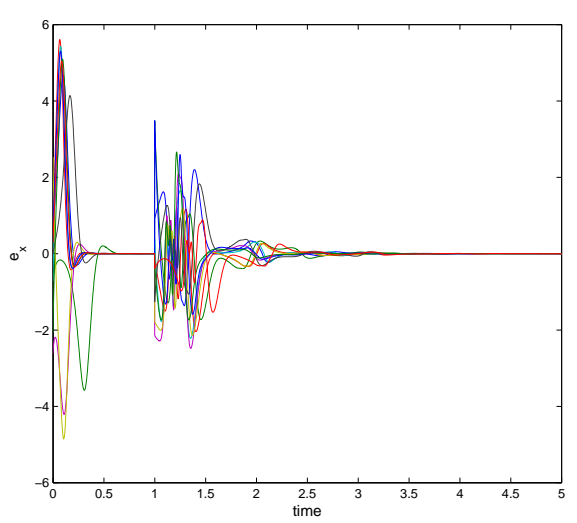

(a)

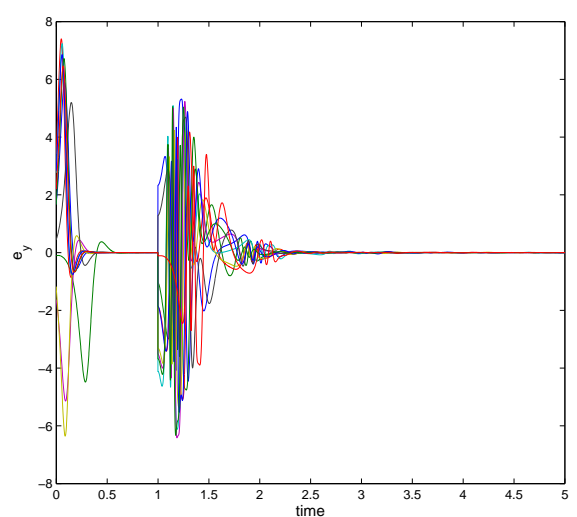

(b)

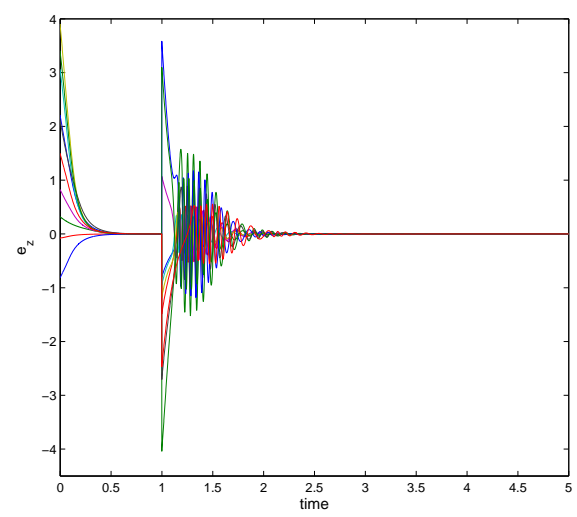

(c)

Figure 3: Time evolution of the lag synchronization error with constant delay coupling.

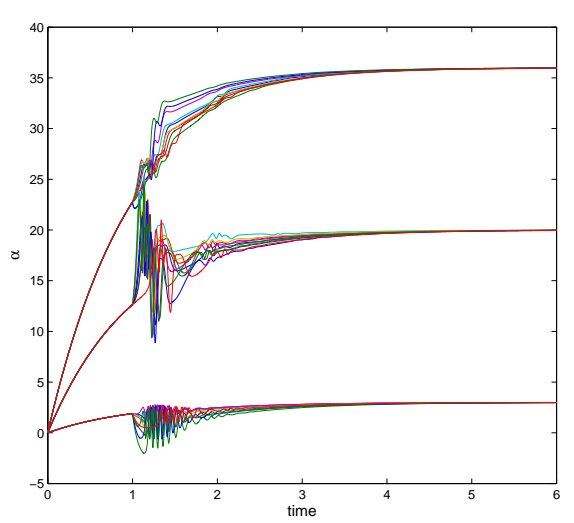

(a)

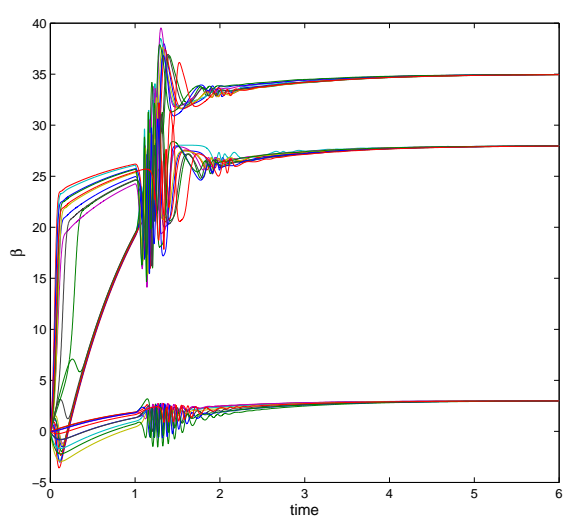

(b)

Figure 4: The estimated unknown parameter of $(a): \hat{\alpha} ;(b): \hat{\beta}$.

\section{Conclusion}

In this paper, we explored a general uncertain complex dynamical networks model with time varying delayed coupling and derivative coupling delay. The adaptive lag synchronization method was studied between uncertain complex dynamical networks with different nodes. Based on the Lyapunov stability theory and adaptive control, lag synchronization criterion was obtained and the unknown parameters 
were identified. The numerical simulation results showed the efficiency of the proposed method.

\section{Acknowledgment}

This work is supported by the UKM research grant DIP-2017-011 and Ministry of Education, Malaysia grant FRGS/1/2017/STG06/UKM/01/1.

\section{References}

[1] A. Abdurahman, H. J. Jiang, Z. D. Teng, Exponential lag synchronization for memristor-based neural networks with mixed time delays via hybrid switching control, J. Franklin Inst., 353 (2016), 2859-2880. 1

[2] G. Al-Mahbashi, M. S. M. Noorani, S. Abu Bakar, S. Vahedi, Adaptive projective lag synchronization of uncertain complex dynamical networks with disturbance, Neurocomputing, 207 (2016), 645-652. 1

[3] S. Boccaletti, V. Latora, Y. Moreno, M. Chavez, D.-U. Hwang, Complex networks: Structure and dynamics, Phys. Rep., 424 (2006), 175-308. 1

[4] R. Cheng, M. Peng, Adaptive synchronization for complex networks with probabilistic time-varying delays, J. Franklin Inst., 353 (2016), 5099-5120. 1

[5] L. Deng, Z. Wu, Q. Wu, Pinning synchronization of complex network with non-derivative and derivative coupling, Nonlinear Dynam., 73 (2013), 775-782. 1, 2.2

[6] H. Du, Function projective synchronization in complex dynamical networks with or without external disturbances via error feedback control, Neurocomputing, 173 (2016), 1443-1449. 1, 2.3

[7] I. Fischer, R. Vicente, J. M. Buldú, M. Peil, C. R. Mirasso, M. C. Torrent, J. Garca-Ojalvo, Zero-lag long-range synchronization via dynamical relaying, Phys. Rev. Lett., 97 (2006), 4 pages. 1

[8] D. H. Ji, S. C. Jeong, J. H. Park, S. M. Lee, S. C. Won, Adaptive lag synchronization for uncertain complex dynamical network with delayed coupling, Appl. Math. Comput., 218 (2012), 4872-4880. 1, 3.2

[9] X. Jian, Y. H. Yang, J. S. Long, Synchronisation of complex networks with derivative coupling via adaptive control, Internat. J. Systems Sci., 44 (2013), 2183-2189. 1, 2.1

[10] Y. Kuang, Delay differential equations: with applications in population dynamics, Academic Press, Boston, (1993). 1

[11] B. C. Li, Pinning adaptive hybrid synchronization of two general complex dynamical networks with mixed coupling, Appl. Math. Model., 40 (2016), 2983-2998. 1

[12] Z. Li, J. A. Fang, T. Huang, Q. Miao, H. Wang, Impulsive synchronization of discrete-time networked oscillators with partial input saturation, Infor. Sci., 422 (2018), 531-541. 1

[13] C. D. Li, X. F. Liao, K.-W. Wong, Chaotic lag synchronization of coupled time-delayed systems and its applications in secure communication, Phys. D, 194 (2004), 187-202. 1

[14] N. Li, H. Sun, Z. Li, Q. Zhang, Adaptive semi-periodically intermittent and lag synchronization control of neural networks with mixed delays, IEEE ACCESS, 6 (2018), 4742-4749. 1

[15] H. M. Liu, W. G. Sun, G. Al-mahbashi, Parameter identification based on lag synchronization via hybrid feedback control in uncertain drive-response dynamical networks, Adv. Difference Equ., 2017 (2017), 11 pages. 1

[16] Y. Liu, Z. Wang, J. Liang, X. Liu, Synchronization and state estimation for discrete-time complex networks with distributed delays, IEEE Trans. Syst. Man Cyber. Part B (Cybernetics), 38 (2008), 1314-1325. 1, 2.4

[17] Z. C. Liu, W. S. Wong, H. Cheng, Cluster synchronization of coupled systems with nonidentical linear dynamics, Internat. J. Robust Nonlinear Control, 27 (2017), 1462-1479. 1

[18] S. A. Pandit, R. E. Amritkar, Characterization and control of small-world networks, Phys. Rev. E, 60 (1999), 1119-1122. 1

[19] L. Shi, H. Zhu, S. Zhong, K. Shi, J. Cheng, Cluster synchronization of linearly coupled complex networks via linear and adaptive feedback pinning controls, Nonlinear Dynam., 88 (2017), 859-870. 1

[20] S. H. Strogatz, Exploring complex networks, Nature, 410 (2001), 268-276. 1

[21] Z. Tang, J. H. Park, J. Feng, Impulsive effects on quasi-synchronization of neural networks with parameter mismatches and time-varying delay, IEEE Trans. Neural Networks Learning Syst., 29 (2018), 908-919. 1

[22] Z. Tang, J. H. Park, T. H. Lee, J. W. Feng, Random adaptive control for cluster synchronization of complex networks with distinct communities, Internat. J. Adapt. Control Signal Process., 30 (2016), 534-549. 1

[23] J.-A. Wang, X. H. Ma, X. Y. Wen, Q. L. Sun, Pinning lag synchronization of driveresponse complex networks via intermittent control with two different switched periods, Phys. A, 461 (2016), 278-287. 1

[24] X. Wang, K. She, S. Zhong, H. Yang, Lag synchronization analysis of general complex networks with multiple time-varying delays via pinning control strategy, Neural Comput. Appl., 2017 (2017), 1-11. 1

[25] Y. Wu, L. Liu, Exponential outer synchronization between two uncertain time-varying complex networks with nonlinear coupling, Entropy, 17 (2015), 3097-3109. 1

[26] Y. H. Xu, W. N. Zhou, J. A. Fang, W. Sun, Adaptive synchronization of the complex dynamical network with nonderivative and derivative coupling, Phys. Lett. A, 374 (2010) 1673-1677. 1 
[27] Y. H. Xu, W. N. Zhou, J. A. Fang, W. Sun, L. Pan, Topology identification and adaptive synchronization of uncertain complex networks with non-derivative and derivative coupling, J. Franklin Inst., 347 (2010), 1566-1576. 1, 2.2

[28] Y. H. Xu, W. N. Zhou, J. A. Fang, C. R. Xie, D. B. Tong, Finite-time synchronization of the complex dynamical network with non-derivative and derivative coupling, Neurocomputing, 173 (2016), 1356-1361. 1

[29] X. S. Yang, Y. M. Feng, K. F. Cedric, Q. Song, F. E. Alsaadi, Synchronization of coupled neural networks with infinitetime distributed delays via quantized intermittent pinning control, Nonlinear Dynam., 94 (2018), 2289-?2303. 1

[30] S. Zheng, Pinning and impulsive synchronization control of complex dynamical networks with non-derivative and derivative coupling, J. Franklin Inst., 354 (2017), 6341-6363. 1, 2.2

[31] P. P. Zhou, S. M. Cai, Adaptive exponential lag synchronization for neural networks with mixed delays via intermittent control, Adv. Difference Equ., 2018 (2018), 17 pages. 1 